\title{
$\mathrm{M}|\mathrm{R}| \mathrm{S}$ Internet Journal Nitride Semiconductor Research
}

\section{New Technique for Sublimation Growth of AIN Single Crystals}

\author{
Y. Shi ${ }^{1}$, B. Liu ${ }^{1}$, Lianghong Liu ${ }^{1}$, J.H. Edgar ${ }^{1}$, E.A. Payzant ${ }^{2}$, J. M. Hayes ${ }^{3}$ and Martin Kuball ${ }^{3}$ \\ ${ }^{1}$ Kansas State University, Department of Chemical Engineering, \\ ${ }^{2}$ Oak Ridge National Laboratory, High Temperature Materials Laboratory, \\ ${ }^{3}$ University of Bristol, H. H. Wills Physics Laboratory,
}

(Received Thursday, January 11, 2001; accepted Sunday, March 4, 2001)

Single crystalline platelets of aluminum nitride (AIN) were successfully grown by a new technique. It consists of (1) depositing an AlN buffer layer on a SiC substrate by metal organic chemical vapor deposition (MOCVD) below $1100^{\circ} \mathrm{C},(2)$ forming an $(\mathrm{AlN})_{\mathrm{x}}(\mathrm{SiC})_{1-\mathrm{x}}$ alloy film on the AlN film by condensing vapors sublimated at a temperature of $1800^{\circ} \mathrm{C}$ from a source mixture of AlN-SiC powders, followed by (3) condensing vapors sublimated from a pure AlN source (at $1800^{\circ} \mathrm{C}$ ). The necessity of the first two steps for the successful AlN sublimation growth on SiC substrate was illustrated by the initial nucleation studies of alloys on SiC substrates with and without MOCVD AIN buffer layers: an AIN MOCVD buffer layer leads to continuous, single grain growth mode; The $(\mathrm{AIN})_{\mathrm{X}}(\mathrm{SiC})_{1-\mathrm{x}}$ alloy film reduces the crack density because its thermal expansion coefficient is intermediate between SiC and AIN. X-ray diffraction (XRD) and Raman spectroscopy studies indicated the high quality of the AlN single crystal.

\section{Introduction}

Despite the rapid progress made in group III nitride based semiconductor film growth [1], better substrates are still needed for high quality epitaxial growth. Currently, there is worldwide interest in producing highquality single crystals suitable for nitride based electronic and optoelectronic devices. The ideal substrate should be lattice matched, isomorphic, and thermal expansion coefficient matched to the film as much as possible. Single crystal AlN is attractive as a substrate for group III-nitride epitaxial growth due to its relatively small lattice constant mismatch along the a-axis $(-2.5 \%)$ with GaN, good thermal stability (melting point $>2500$ ${ }^{\circ} \mathrm{C}$ ), high resistivity and similar coefficient of thermal expansion [2]. To date, bulk AlN single crystals are limited in sizes to diameters below $15 \mathrm{~mm}$ [3].

Bulk AlN crystals are most commonly produced by vapor transport (sublimation) from an AIN source. This technique was most successfully developed by Slack and McNelly [4], [5] in the mid-1970's. Large AlN single crystals, up to $1 \mathrm{~cm}$ long and $0.4 \mathrm{~cm}$ in diameter, were grown by self-seeding from the sharp tip of sealed tungsten crucibles heated in an radio frequency induction furnace. Recently, Schowalter et al [3] demonstrated good quality AlGaN epitaxy on bulk AlN crystals produced by self-seeding.
Seeding bulk AIN growth on silicon carbide substrates instead of self-seeding offers several potential advantages including the availability of large diameter seed crystals (up to $100 \mathrm{~mm}$ ) for rapid process scale-up, and better control of the crystal polarity, through the choice of the $\mathrm{SiC}$ seed crystal polarity (C or Si polarity). Balkas et al [6] first produced thick ( $\leq 1 \mathrm{~mm})$ AlN films on $10 \times 10 \mathrm{~mm}^{2} 6 \mathrm{H}-\mathrm{SiC}$ substrates in a resistively heated graphite furnace. Their results presented three problems with $6 \mathrm{H}-\mathrm{SiC}$ as seed crystals: the decomposition of the $\mathrm{SiC}$ substrate at high crystal growth temperatures, the formation of individual grains in the AlN crystal, and cracking of AlN due to differences in the thermal expansion coefficients of AlN and $\mathrm{SiC}$.

The author's previous study of pure AlN grown on $\mathrm{SiC}$ substrate showed that direct growth on Si-polarity as-received $\mathrm{SiC}$ substrates was discontinuous, marked by sparse nucleation and slow lateral growth of nuclei [7]. Multiple hexagonal AIN grains typically formed after a long growth time (more than 3 hours) due to nucleation at several sites and island growth mode. A technique for suppressing $\mathrm{SiC}$ decomposition and grain boundary formation in the AlN films was developed by first depositing an AIN buffer layer by MOCVD before sublimation growth [7]. 
The current paper describes a solution for a third problem: cracks. The tensile stress due to thermal expansion coefficient mismatch between the AlN layer and the $\mathrm{SiC}$ substrate caused cracking during cooling. Cracks are effectively reduced by forming an $(\mathrm{AlN})_{\mathrm{x}}(\mathrm{SiC})_{1-\mathrm{x}}$ alloy on the $\mathrm{SiC}$ substrate with AlN MOCVD buffer layer, then depositing pure AlN on the alloy seed by sublimation because its thermal expansion coefficient is intermediate between $\mathrm{SiC}$ and $\mathrm{AlN}$, hence the thermally generated stress can be reduced. Besides, the presence of silicon and carbon in the source material reduces or eliminates the decomposition of the $\mathrm{SiC}$ substrate during sublimation crystal growth, enabling longer duration crystal growth.

$(\mathrm{AlN})_{\mathrm{x}}(\mathrm{SiC})_{1-\mathrm{x}}$ alloys are of interest not only as the seeds for pure AlN single crystal growth, but also as promising semiconductors themselves because the physical and electronic properties can be controlled by selecting a specific AlN:SiC alloy composition. The research group at the University of Dagestan extensively investigated the properties of epitaxial $(\mathrm{AlN})_{\mathrm{x}}(\mathrm{SiC})_{1-\mathrm{x}}$ films grown by the sublimation method on $6 \mathrm{H}-\mathrm{SiC}$ substrates from sintered polycrystalline $\mathrm{SiC}$ AlN sources in argon and nitrogen mixtures through the full compositional range $x$ [8], [9], [10]. The energy band gap can be controlled by the alloy composition over a very wide range (from $2.9 \mathrm{eV}$ for pure $\mathrm{SiC}$ to 6.2 $\mathrm{eV}$ for pure $\mathrm{AlN}$ ), the band transition is direct for $\mathrm{x}>0.7$ and both $\mathrm{n}$ - and p-type conductivity are possible. In reference [8], the composition of the epitaxial $(\mathrm{AlN})_{\mathrm{x}}(\mathrm{SiC})_{1-\mathrm{x}}$ was measured to have a linear relationship with the source materials (only for aluminum however) but no detailed experimental data was provided to support this relationship. A recently approved patent reported the growth of bulk $(\mathrm{AlN})_{0.7}(\mathrm{SiC})_{0.3}$ single crystals 2 inches in diameter and $46-50 \mathrm{~mm}$ thick by the same technique from a polycrystalline $(\mathrm{AlN})_{0.7}(\mathrm{SiC})_{0.3}$ source. The chemical composition analysis of the grown crystal was not reported, only the nominal composition $(\mathrm{AlN})_{0.7}(\mathrm{SiC})_{0.3}$ of the source. The crystal quality characterization was also not reported in this patent.

All the previous studies of $(\mathrm{AlN})_{\mathrm{x}}(\mathrm{SiC})_{1-\mathrm{x}}$ alloy growth used only as-received $\mathrm{SiC}$ substrates without MOCVD buffer layers; they used polycrystalline sources in contrast to our use of only mechanical mixture of AlN and $\mathrm{SiC}$ powders [8], . The structural investigations reported in Ref. [8] show the morphologies and structural qualities of the heteroepitaxial layers deteriorating with the AlN content. When $\mathrm{x} \leq 0.5$, the perfection of the epitaxial layers of $(\mathrm{SiC})_{1-\mathrm{x}}(\mathrm{AlN})_{\mathrm{x}}$ was comparable with that of the $\mathrm{SiC}$ substrate. Increasing $\mathrm{x}$ $(x \geq 0.5)$ deteriorated the morphologies of the epitaxial layers and caused the appearance of a mosaic structure. On using a 90wt $\%$ AlN source, heterogeneous growth of AlN on SiC was observed. Patent reported large single crystals grown from $(\mathrm{AlN})_{0.7}(\mathrm{SiC})_{0.3}$ polycrystalline source on a bare $\mathrm{SiC}$ substrate. However, we found that AlN single crystals could not be produced on bare $\mathrm{SiC}$ substrates in our furnace using the growth parameters given in Ref. .

In this paper, a new technique employing $\mathrm{SiC}$ single crystals as substrates for bulk crystal growth of AlN by the sublimation method was developed. The method consists of (1) depositing an AlN buffer layer on the SiC substrate by metal organic chemical vapor deposition (MOCVD) below $1100^{\circ} \mathrm{C},(2)$ forming an $(\mathrm{AIN})_{\mathrm{x}}(\mathrm{SiC})_{1-}$ ${ }_{x}$ alloy film on the above AlN film by condensing vapors sublimated at a temperature of $1800^{\circ} \mathrm{C}$ from a source mixture of AlN-SiC powders, followed by (3) condensing vapors sublimated from a pure AlN source (at $1800^{\circ} \mathrm{C}$ ). How the first two steps help solve the three problems for the AlN sublimation growth on $\mathrm{SiC}$ substrate is illustrated by initial nucleation studies of alloys on $\mathrm{SiC}$ substrates with and without MOCVD buffer layers. The high quality AlN crystal obtained from the above three steps was characterized by SAM (Scanning Auger Microscopy), Raman scattering and X-ray diffraction techniques.

\section{Experimental procedures}

\subsection{Crystal growth}

Sublimation growth experiments were conducted in a resistively heated furnace using tungsten wire mesh heating elements. The seed and source were enclosed in two concentric tungsten crucibles to contain the $\mathrm{Al}$ vapor as it will seriously damage the tungsten heating elements. The source materials are AlN (99.5\%, Tokuyama Soda Co., LTD, Japan) and SiC powder (99.8\%, Alfa Aesar, USA). The source materials for alloy growth with proper $\mathrm{AlN}: \mathrm{SiC}$ ratio were thoroughly mixed in an alumina mortar and sintered at $1800^{\circ} \mathrm{C}$ and 500 torr in the same furnace before sublimation growth. The $\theta-2 \theta$ x-ray diffraction pattern of source materials after 60 hours sintering still showed the mixture of AlN and $\mathrm{SiC}$ powders instead of polycrystalline $(\mathrm{AlN})_{\mathrm{x}}(\mathrm{SiC})_{1-\mathrm{x}}$ alloys. The seed holders were sintered AlN with approximately $1 \%$ oxygen as the main impurity as analyzed by a standard inert gas fusion method (TC-136, Leco Co.). The as-received (0001) 6H-SiC silicon polarity on-axis wafers were cut into about $2 \mathrm{~cm} \times 2 \mathrm{~cm}$ substrates. All the substrates were ultrasonically degreased in organic solvents, rinsed in deionized water, and blown in dry nitrogen before loading into the furnace. The distance between the source and the substrate was kept constant at $2 \mathrm{~mm}$. The backside of the 
seed was covered by a sintered AlN cap to reduce $\mathrm{SiC}$ dissociation.

Information about the furnace and growth process has been described in detail in Ref. [7]. The growth ambient was $99.9999 \%$ pure nitrogen, and the background pressure during growth was 500 torr. The in-situ growth temperature was measured by an optical pyrometer focused on the top lid of the outside crucible. According to the pre-measured temperature profile, at our experimental condition the source temperature was about $1800^{\circ} \mathrm{C}$ and the source-substrate temperature difference was about $10^{\circ} \mathrm{C}$ for $2 \mathrm{~mm}$ source-substrate distance. The growth temperature was kept constant for all growths by fixing the furnace output power during the crystal growth so the results could be fairly compared.

The thickness of samples from lengthy growths was measured with a micrometer (the accuracy is about $1 \mu \mathrm{m})$. The average growth rate was about $5 \mu \mathrm{m} / \mathrm{hr}$ at $1800^{\circ} \mathrm{C}$ and 500 torr. Therefore, the nominal thickness of the samples grown at the same experimental condition for 15,45 and 120 minutes was $1.25,3.75$ and 10 $\mu \mathrm{m}$, respectively, assuming constant growth rates for the whole growth process.

\subsection{Buffer layer preparation by MOCVD}

The AlN buffer layer was grown in a vertical MOCVD reactor at low pressure (76 torr). Before loading into the reactor, the $6 \mathrm{H}-\mathrm{SiC}$ substrates were cleaned using the same standard cleaning procedure as described above. Trimethylaluminum (TMA) and ammonia $\left(\mathrm{NH}_{3}\right)$ were the $\mathrm{Al}$ and $\mathrm{N}$ sources, and Pd-cell purified $\mathrm{H}_{2}$ was the carrier gas. The substrates were preheated at $1100{ }^{\circ} \mathrm{C}$ for 10 minutes in $3 \mathrm{slm}$ of $\mathrm{H}_{2}$ for surface cleaning. Then the AlN films were deposited at the same temperature under $\mathrm{H}_{2}, \mathrm{NH}_{3}$ and TMA flow rates of $3 \mathrm{slm}, 3 \mathrm{slm}$ and 30 sccm, respectively. An approximate $2 \mu \mathrm{m}$ thick AlN buffer layer was obtained for the 4 hour growth.

\subsection{Characterization}

The as-grown AlN crystals were first characterized by Nomarski differential interference contrast microscopy (NDIC) at magnifications of 60X.

The relative chemical composition was characterized by scanning Auger microscopy (SAM) using a Physical Electronics PHI680 Auger Nanoprobe operated at a less than optimal $3 \mathrm{kV}$ or less because of the charging problem. To ensure the accuracy of composition measurement, samples were sputtered by argon ion for several minutes until oxygen could not be detected.

$\mathrm{X}$-ray diffraction measurements were conducted using a Scintag PTS four-axis goniometer diffractometer with $\mathrm{Cr} \mathrm{K} \alpha$ radiation $(25 \mathrm{kV} \times 8 \mathrm{~mA})$ and a high purity germanium solid state detector. The lattice parameters and crystal qualities were analyzed by $\mathrm{x}$-ray diffraction
(XRD) of both symmetric (0002) and asymmetric $(11 \overline{2} 2)$ planes including conventional $\theta-2 \theta$ mode, $\theta$ mode (i.e., rocking curve), and pole figures. The pole figures were obtained by tilting the normal of the sample out of the x-ray plane defined by the source and detector to an angle $\psi$ which was varied from $0^{\circ}$ to $10^{\circ}$ in $0.5^{\circ}$ increments. Then the diffracted intensities were measured for the special (0002) or (112̄2) planes as the sample was rotated about the normal through an angle $\phi$ from $0^{\circ}$ to $360^{\circ}$. Pole figures represent the texture of a material by showing the distribution of particular crystallographic directions (poles =directions normal to planes) in a stereographic projection. The density of the poles is represented in the pole figure by equal density contour levels. A small misalignment of the samples causes a large loss in the diffraction intensity from the crystals. Thus before $\mathrm{x}$-ray measurement $(\theta-2 \theta$ and $\theta$ mode), the samples always were aligned exactly by searching the right $\psi$ (tilt) and $\phi$ (rotation) positions from the pole figure where the maximum diffraction intensity was collected. The size of incident beam collimators was gradually decreased from $1 \mathrm{~mm}$ to $0.1 \mathrm{~mm}$ until the measured FWHM (full width at half maximum) of the rocking curve didn't change with decreasing collimator size, so that the FWHM was representative of the sample rather than the instrument. Based on this test, a $0.3 \mathrm{~mm}$ incident beam collimator was used for all the subsequent measurements.

Micro-Raman spectra were obtained using a Renishaw UV micro-Raman system with the $325 \mathrm{~nm}$ line of a $\mathrm{HeCd}$ laser as the excitation source. The spot size and the spectral resolution were $1-2 \mu \mathrm{m}$ and $3-4 \mathrm{~cm}^{-1}$, respectively.

\section{Results}

\subsection{Nucleation studies of (AIN $)_{x}(\mathrm{SiC})_{1-x}$ alloys on $6 \mathrm{H}-\mathrm{SiC}$ substrates}

The initial nucleation of the alloy on the $\mathrm{SiC}$ substrates with AIN MOCVD buffer layers was continuous and smooth. In contrast, alloy films grown on bare SiC substrates under our experimental conditions produced only discontinuous films regardless of the source $\mathrm{AlN}: \mathrm{SiC}$ ratio. To make the experimental results comparable, 1:1 molar ratio mixtures of $\mathrm{AlN}$ and $\mathrm{SiC}$ powder sources were deposited on two $\mathrm{SiC}$ substrates, one with and one without AlN MOCVD buffer layers during the same growth run with growth times of 15, 45, 120 minutes and 100 hours, respectively. Their optical micrographs are shown in Figure 1 denoted by (1), (2), (3) and (4) in the sequence of the growth time increase.

On bare $\mathrm{SiC}$ substrates, the surface morphology became coarser with increasing growth time, and the isolated alloy nuclei did not coalesce, similar to the case of pure AlN growth on bare $\mathrm{SiC}$ substrates [7]. Figure 
1-a(1-4) are the optical micrographs of alloy crystals grown on bare $\mathrm{SiC}$ substrates. After 15 minutes growth, the majority of the substrate surfaces were very smooth and featureless, except for a few tiny black dots, possibly from the alloy nucleation $(\mathrm{a}(1))$. Sparse hexagonal alloy nuclei formed after 45 minutes growth $(\mathrm{a}(2))$. After 120 minutes of growth, both the size and density of alloy nuclei increased (a(3)). After 100 hours of growth, an alloy film about $0.5 \mathrm{~mm}$ thick but still discontinuous was produced as shown in Figure 1-a(4).

In contrast, the surface morphology of $(\mathrm{AlN})_{\mathrm{x}}(\mathrm{SiC})_{1-}$ $x$ alloys grown on $\mathrm{SiC}$ substrates with MOCVD buffer layers became smooth and continuous with longer growth times as shown in an optical micrographs in Figure 1-b(1-4). The sample grown for 15 minutes had numerous hexagonal nuclei, some of which had already coalesced. Cracks were observed due to different thermal expansion coefficients between the alloy and the substrate. After 45 minutes growth all the small hexagonal nuclei merged together forming large hexagons with cracks distributing in the whole growth surface. A 2 hour sample showed relatively smooth surface with macro-growth steps. The crack density was significantly reduced. Apparently, the threshold for cracking from tensile stress caused by thermal expansion difference increased as the thickness of the growth film increased. Growth for 100 hours produced a continuous $0.5 \mathrm{~mm}$ thick alloy film. In most cases, the alloy crystals still peeled off from the substrate and cracked into several pieces due to the thermal stress on cooling. The crackfree areas were much larger (about $1 \mathrm{~cm}^{2}$ ) than for AlN crystals grown without the alloy layer (usually < $0.5 \mathrm{~cm}^{2}$ ). The alloy growth surface was very smooth and featureless; the irregular shadow shown in Figure 1-b(4) originates from the reflection from a peeling-surface.

The alloy film had the same composition, approximately $(\mathrm{AlN})_{0.8}(\mathrm{SiC})_{0.2}$, regardless of the ratio of AlN to $\mathrm{SiC}$ powder in the source. Alloy film were produced from three different $\mathrm{AlN}: \mathrm{SiC}$ ratios in the source 1:1, $1: 2$, and $1: 3$, at $1850^{\circ} \mathrm{C}$ and 500 torr of $\mathrm{N}_{2}$. For all three cases, the alloy composition was nearly identical as measured by Scanning Auger Microscopy (SAM). This can be explained by considering the partial pressure of the components produced by the source materials and determined by temperature and pressure, which are in turn responsible for producing the alloy films. As long as there exists at least some solid phase of each source compound, the partial pressures of the volatile components will not change. Adding more of either source compounds does not change the composition of the gas phase.

\subsection{Pure AIN growth and Characterization}

Using an $(\mathrm{AlN})_{0.8}(\mathrm{SiC})_{0.2}$ alloy single crystal as the seed, pure AlN was sublimated to form a high quality AlN single crystal at $1800^{\circ} \mathrm{C}, 500$ torr for 100 hours. Figure 2 is the optical micrograph of AlN about $6 \times 4 \mathrm{~mm}$ large and $0.5 \mathrm{~mm}$ thick. The crystal was light yellow and free of cracks with a very smooth and featureless growth surface.

The conventional $\theta-2 \theta$ diffraction patterns of sym-

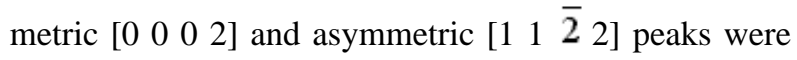
measured for the AlN single crystal. The lattice parameters determined from these two peaks were $a=3.113$ and $\mathrm{c}=4.996 \AA$ in agreement with the reported lattice parameters of AlN [12]. The rocking curves FWHM (full width at half maximum) and peak positions are reported in Table 1. For comparison, data obtained for the $\mathrm{SiC}$ substrate and $(\mathrm{AlN})_{0.8}(\mathrm{SiC})_{0.2}$ alloy seed are also presented in the same table. The AlN single crystal has [0 0

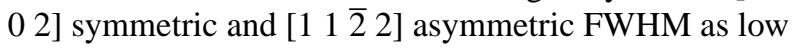
as 238 and 461 arcsec, respectively. Although the value

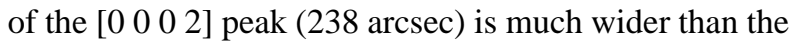

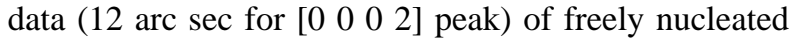
AlN small crystal by sublimation method in reference [13], insufficient experimental details were reported in that reference to compare the two data. Under the same $\mathrm{X}$-ray analysis condition, the [0 [ $\left.\begin{array}{lll}0 & 0 & 2\end{array}\right]$ and $\left[\begin{array}{llll}1 & 1 & \overline{2} & 2\end{array}\right]$ peaks rocking curve $\mathrm{FWHM}$ for the $\mathrm{SiC}$ substrate were 489 and 562 arcsec, suggesting the AlN single crystal was of comparable quality to the original substrate. The alloy crystal had a much wider rocking curve FWHM values for both symmetric and asymmetric peaks (1141 and 1454 arcsec, respectively) compared to either the asreceived $\mathrm{SiC}$ substrate and the pure AlN single crystal. This is not unexpected because of the atomic occupancy disorder in the alloy crystal shown by four-circle diffraction data of a single crystal and composition variation along the growth axis that was measured by Scanning Auger Microscopy (SAM) and will be published elsewhere. Apparently, pure AlN sublimation growth on the alloy seed improved the crystal quality significantly. The disparity between the FWHM of the symmetric and asymmetric peaks is commonly observed for nitride growth [14], [15], [16] and has been attributed to the presence in the nitride films of significantly more dislocations of edge type rather than screw type [16], [17]. The pole figures of $\left[\begin{array}{llll}0 & 0 & 0 & 2\end{array}\right]$ and $\left[\begin{array}{llll}1 & 1 & \overline{2} & 2\end{array}\right]$ peaks of AlN single crystal were measured and are shown in Figure 3. Such clean pole figures indicate that the AlN crystal has very good quality.

A Raman spectrum recorded on the (0001) face of the AlN single crystal is presented in Figure 4. All the peaks can be assigned to AIN Raman modes: 
$\mathrm{A}_{1}(\mathrm{LO}) \approx 889 \mathrm{~cm}^{-1}$ and $\mathrm{E}_{2}$ (high) $\approx 655 \mathrm{~cm}^{-1}$. The linewidth of $\mathrm{E}_{2}$ mode was as narrow as $9 \mathrm{~cm}^{-1}$ which also verified the high quality of our crystal [18]. The AlN phonon frequencies point to a largely unstrained AlN crystal in agreement with XRD results [19], [20].

\section{Conclusions}

Seeded bulk growth of a crack-free AlN single crystal was achieved on a $\mathrm{SiC}$ substrate by (1) depositing an AlN buffer layer on the $\mathrm{SiC}$ substrate by MOCVD below $1100^{\circ} \mathrm{C},(2)$ forming an $(\mathrm{AlN})_{\mathrm{X}}(\mathrm{SiC})_{1-\mathrm{x}}$ alloy film by condensing vapors sublimated (at temperatures above $1700^{\circ} \mathrm{C}$ ) from a source mixture of AlN-SiC powders, followed by (3) condensing vapors sublimated from a pure AlN source. This method solves three problems previously associated with using $\mathrm{SiC}$ as seed crystals for AlN growth. First, the low temperature deposited AlN layer promotes layer-by-layer growth forming a continuous single grain. Second, the intermediate properties of the $(\mathrm{AlN})_{\mathrm{x}}(\mathrm{SiC})_{1-\mathrm{x}}$ alloy reduces cracking in the growing crystal. Third, the presence of silicon and carbon in the source material reduces or eliminates the decomposition of the $\mathrm{SiC}$ substrate during sublimation crystal growth, enabling longer duration crystal growth. By employing this technique, a high quality AlN single crystal was produced as determined by Raman spectroscopy and X-ray diffraction. Such crystals are ideal for seeding AlN crystal growth at high temperature and hence high growth rates, to produce substrates for $\mathrm{Al}_{\mathrm{x}} \mathrm{Ga}_{1-\mathrm{x}} \mathrm{N}$ based electronics.

\section{ACKNOWLEDGMENTS}

We are grateful for the support of this research from BMDO (Contract No: N00014-98-C-0407), ONR (Contract No. N00014-99-1-0104), and the Assistant Secretary for Energy Efficiency and Renewable Energy, Office of Transportation Technologies, as part of the High Temperature Materials Laboratory User Program, Oak Ridge National Laboratory, managed by UT-Battelle, LLC, for the U.S. Department of Energy under contract number DEAC05-00OR22725. The work at the University of Bristol was supported by Renishaw plc, EPSRC, the Royal Society and British Council.

\section{REFERENCES}

[1] S. Nakamura, M. Senoh, N. Iwasa, S. Nagahama, Jpn. J. Appl. Phys. 34, 797 (1995).

[2] H. Q. Lu, I. B. Bhat, B. C. Lee, G. A. Slack, L. J. Schowalter, Mater. Res. Soc. Symp. Proc. 482, 277 (1997).

[3] L. J. Schowalter, Y. Shusterman, R. Wang, I. Bhat, G. Arunmozhi, G. A. Slack, Appl. Phys. Lett. 76, 985 (2000).

[4] G. A. Slack, T. F. McNelly, J. Cryst. Growth 34, 263 (1976).

[5] G. A. Slack, T. F. McNelly, J. Cryst. Growth 42, 560 (1977).

[6] C. M. Balkas, Z. Sitar, T. Zheleva, L. Bergman, R. Nemanich, R. F. Davis, J. Cryst. Growth 179, 363 (1997).

[7] Y. Shi, Z. Y. Xie, L. H. Liu, B. Liu, J. H. Edgar, M. Kuball,"Influence of Buffer Layer and 6H-SiC Substrate Polarity on the Nucleationof AlN Grown by the Sublimation Sandwich Technique", J. Cryst. Growth, (2001) in press.

[8] G. K. Safaraliev, Yu. M. Tairov, V. F. Tsvetkov, Sov. Phys. Semicond. 25, 865 (1991).

[9] G. K. Safaraliev, G. K. Sukhanek, Yu. M. Tairov, V. F. Tsvetkov, Izv. Akad. Nauk. SSR Inorg. Mater. 22, 1839 (1986).

[10] N. V. Ofitservoa, M. K. Kurbanov, I. P. Nikitina, N. D. Sorokin, G. K. Safaraliev, Yu. M. Tairov, V. F. Tsvetkov, Izv. Akad. Nauk. SSR Inorg. Mater. 28, 2011 (1991).

[11] C. E. Hunter, Growth of Bulk Single Crystals of Aluminum Nitride: Silicon Carbide Alloys, Patent Number 6086672, United States Patent Office (2000).

[12] W. J. Meng, "Crystal structure, mechanical properties, thermal properties and refractive index of AlN" in Properties of Group III Nitrides, edited by J.H. Edgar (INSPEC, London, 1994)

[13] M. Tanaka, S. Nakahata, K. Sogabe, H. Nakata, M. Tobioka, Jpn. J. Appl. Phys. 36, 1062 (1997).

[14] F. A. Ponce, B. S. Krusor, J. S. Major, W. E. Plano, D. F. Welch , Appl. Phys. Lett. 67, 410-412 (1995).

[15] Q. Zhu , A. Botchkarev, W. Kim, O. Aktas, A. Salvador, B. Sverdlov, H. Morkoc, S.-C. Y. Tsen, David J. Smith , Appl. Phys. Lett. 68, 1141-1143 (1996).

[16] B. Heying, X. H. Wu, S. Keller, Y. Li, D. Kapolnek, B. P. Keller, S. P. DenBaars, J. S. Speck, Appl. Phys. Lett. 68, 643-645 (1996).

[17] T. Metzger, R. Stömmer, M. Schuster, H. Göbel, R. Höpler, E. Born, T. H. Metzger, S. Christiansen, H. P. Strunk, O. Ambacher, M. Stutzmann, Phys. Stat. Sol. A 162, 529 (1997).

[18] M. Kuball, J. M. Hayes, Y. Shi, J. H. Edgar, Appl. Phys. Lett. 77, 1958 (2000).

[19] M. Kuball, J. M. Hayes, A. D. Prins, N. W. A. van Uden, D. J. Dunstan, Y. Shi, J. H. Edgar, Jpn. J. Appl. Phys. 39, 710 (2000).

[20] J. M. Hayes, M. Kuball, Y. Shi, J. H. Edgar, Jpn. J. Appl. Phys. 39, 710 (2000). 


\section{FIGURES}
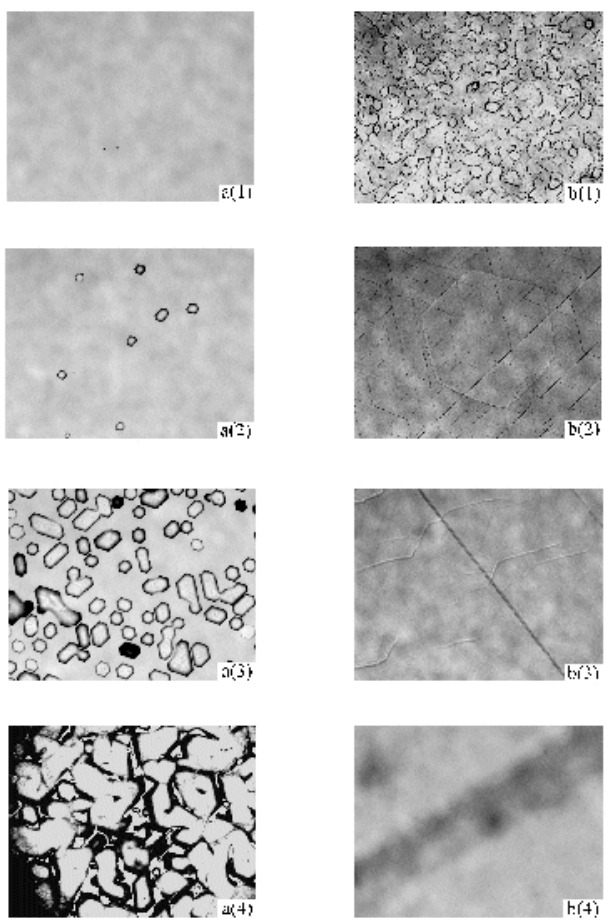

Figure 1. Optical micrographs of $(\mathrm{AlN})_{\mathrm{x}}(\mathrm{SiC})_{1-\mathrm{x}}$ alloy crystal growth with magnification $60 \times$ on: (a) the as-received $\mathrm{SiC}$ substrates, (b) SiC substrates with AlN MOCVD buffer layer; (1-4) denote the growth time sequence of $15,45,120$ minutes and 100 hours.

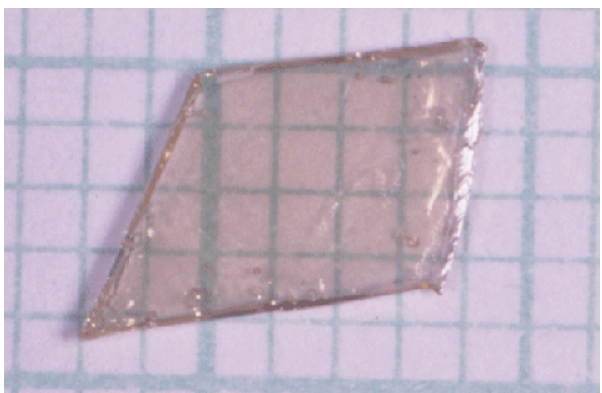

Figure 2. Photograph of pure AlN grown for 100 hours, one grid represents $1 \mathrm{~mm}$.

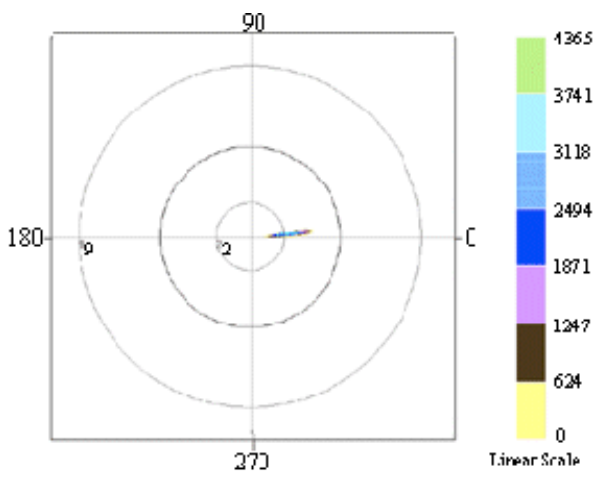

(a)

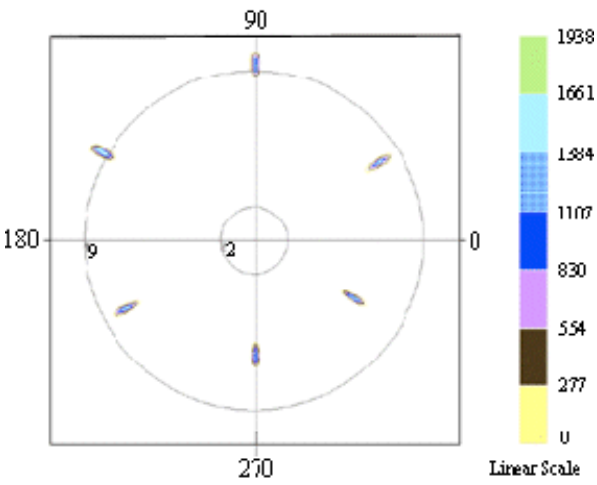

(b)

Figure 3. Pole figure of (a) symmetric (0002), and (b) asymmetric $(11 \overline{2} 2)$ plane for pure AlN crystal.

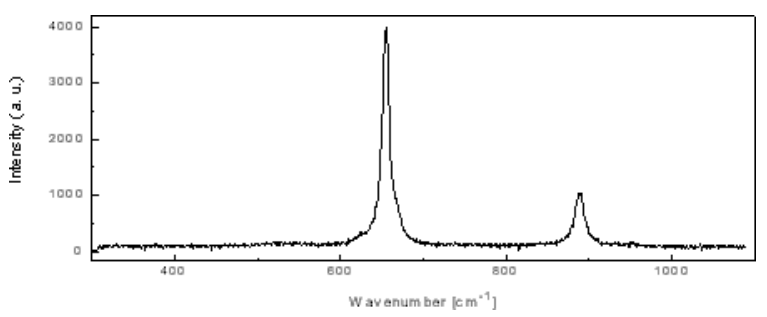

Figure 4. Raman spectra of pure AlN single crystal.

\section{TABLES}

Table 1. Results of x-ray rocking curve of symmetric [ $\left[\begin{array}{llll}0 & 0 & 0 & 2\end{array}\right]$ asymmetric $\left[\begin{array}{llll}1 & 1 & \overline{2} & 2\end{array}\right]$ peaks and lattice parameters of SiC substrate, $(\mathrm{AlN})_{0.8}(\mathrm{SiC})_{0.2}$ alloy and pure AlN crystal.

\begin{tabular}{|c|c|c|c|c|c|c|}
\hline Sample & & 0 2] & & $\overline{2} 2]$ & $\mathrm{a}(\AA)$ & c $(\AA ⿻)$ \\
\hline & $2 \theta(\mathrm{deg})$ & FWHM (arcsec) & $2 \theta(\mathrm{deg})$ & FWHM (arcsec) & & \\
\hline SiC substrate & 53.75 & 489 & 120.93 & 562 & 3.080 & 5.065 \\
\hline$(\mathrm{AIN})_{0.8}(\mathrm{SiC})_{0.2}$ alloy & 54.32 & 1141 & 120.32 & 1454 & 3.104 & 5.016 \\
\hline Pure AIN & 54.56 & 238 & 120.12 & 461 & 3.113 & 4.996 \\
\hline
\end{tabular}

